\title{
Magnetic Resonance Imaging Patterns of Post-Operative Spinal Infection : Relationship between the Clinical Onset of Infection and the Infection Site
}

\author{
Seon-Jeong Kim, M.D., ${ }^{1,2}$ Sang Hoon Lee, M.D., Ph.D., ${ }^{1}$ He Won Chung, M.D., Ph.D., ${ }^{1}$ Min Hee Lee, M.D., Ph.D., ${ }^{1}$ \\ Myung Jin Shin, M.D., Ph.D., Seoung Woo Park, M.D., Ph.D. ${ }^{3}$ \\ Department of Radiology and Research Institute of Radiology, Asan Medical Center, University of Ulsan College of Medicine, Seoul, Korea \\ Department of Radiology, ${ }^{2}$ Myongji Hospital, Seonam University College of Medicine, Goyang, Korea \\ Department of Neurosurgery, ${ }^{3}$ School of Medicine, Kangwon National University, Chuncheon, Korea
}

Objective : To investigate the magnetic resonance imaging (MRI) findings and the patterns of postoperative spinal infection according to the passage of time.

Methods : Institutional review board approval was obtained, and informed consent was not obtained for the retrospective review of patients' medical records. A total of 43 patients (27 men and 16 women; mean age, 64) diagnosed with postoperative spinal infection were included in this study. We retrospectively reviewed the MRI findings and the medical records and categorized the infection sites based on MRI, i.e., anterior, posterior, and both parts. The duration of the clinical onset from surgery was divided, i.e., acute ( $\leq 2$ weeks), subacute ( $2-4$ weeks), and late ( $>4$ weeks).

Results : Postoperative spinal infection was involved in the posterior part in $31(72 \%)$, anterior part in two (4.7\%), and both parts in 10 patients (23.3\%). Abscess or phlegmon in the back muscles and laminectomy site were the most common MRI findings. The number of patients with acute, subacute, and late clinical onset were 35, two, and six, respectively (mean, 33.4 days; range, 1-730 days). The mean duration of the clinical onset was 12 days in the posterior part, 15.2 days in both parts, and 456.5 days in the anterior part.

Conclusion : Postoperative spinal infection usually occurred within four weeks in the posterior part and over time the infection was considered to spread into the anterior part. For the evaluation of postoperative spinal infection, the posterior surgical field was more important than the vertebral body or the disc space on MRI.

Key Words : Magnetic resonance imaging $\cdot$ Spondylitis, Discitis $\cdot$ Surgery $\cdot$ Spine $\cdot$ Infection.

- Received : May 13, $2015 \bullet$ Revised : December 20, $2015 \bullet$ Accepted : April 24, 2016

- Address for reprints : Sang Hoon Lee, M.D., Ph.D.

Department of Radiology and Research Institute of Radiology, Asan Medical Center, University of Ulsan College of Medicine, 88 Olympic-ro 43-gil, Songpa-gu, Seoul 05505 , Korea

Tel : +82-2-3010-3983, Fax : +82-2-476-4719, E-mail : shlee@amc.seoul.kr

This is an Open Access article distributed under the terms of the Creative Commons Attribution Non-Commercial License (http://creativecommons.org/licenses/by-nc/4.0) which permits unrestricted non-commercial use, distribution, and reproduction in any medium, provided the original work is properly cited. 


\section{INTRODUCTION}

Spinal infection is one of the most serious complications of spine surgery and the incidence of infection ranges from $0.7 \%$ to $12 \%{ }^{17)}$. Clinical presentations, laboratory testing, and imaging findings should all be considered for the diagnosis of postoperative spinal infection ${ }^{18)}$. Magnetic resonance imaging (MRI) is the most important imaging modality for evaluating postoperative spinal infection ${ }^{1)}$. To date, several studies have reported the findings of postoperative osteomyelitis, discitis, and epidural abscesses ${ }^{6,7)}$. Boden et al. ${ }^{1)}$ reported differential MRI findings between non-pathologic postoperative changes and discitis during the early postoperative period as well as signal intensity changes in the disc space and adjacent bone marrow on pre- and post-enhancement scans. To our knowledge, information regarding the relationship between the clinical onset of infection and the infection site, as seen on MRI, has rarely been published ${ }^{8}$. Reviewing the MRI of postoperative spinal infection, we suspected that the postoperative infection begin at the manipulation site and then spread to adjacent local structures. Therefore, we performed a retrospective review of the spine MRI results in patients with proven postoperative spinal infection in order to evaluate the relationship between the infection site seen on MRI and the elapsed time to postoperative spinal infection.

\section{MATERIALS AND METHODS}

\section{Study population}

Institutional review board approval was obtained, and informed consent was not obtained for the retrospective review of the patients' medical records. We searched the clinical database for 158 patients with postoperative spinal infection and who underwent spine MRI and surgical or image-guided tissue biopsy/aspiration or blood culture at a single medical institution between January 1993 and March 2012. Patients who did not develop postoperative spinal infection seen on MRI $(\mathrm{n}=96)$, an infection site in the harvest site only $(\mathrm{n}=2)$, negative micro-organism on determined on culture $(\mathrm{n}=11)$ or a previous surgical history of spinal infection $(\mathrm{n}=6)$ were excluded. A total of 43 patients (16 women, 27 men) were included in our study and their mean age was 64 years (range, 34-83 years). We reviewed the MRI findings and the medical records of these patients. The surgical approach and level, clinical symptoms and signs, laboratory findings (erythrocyte sedimentation rate [ESR], C-reactive protein [CRP], and white blood cell [WBC] counts), and causative micro-organism were also reviewed in all patients. The interval between surgery and the onset of clinical symptoms/signs or abnormal laboratory tests was divided into three groups : acute ( $\leq 2$ weeks); subacute ( $2-4$ weeks); and late ( $>4$ weeks). The interval between surgery and MRI was also divided into three groups : acute ( $\leq 2$ weeks); subacute ( $2-4$ weeks); and late ( $>4$ weeks).

\section{MRI}

MRI was performed on 1.5-T units (Intera, Achieva : Philips Healthcare, Best, The Netherlands; Vision, Avanto : Siemens Medical Solutions, Erlangen, Germany). For the lumbar or thoracolumbar spine, MRI was performed using a spinal coil and the following imaging parameters : sagittal T1-weighted imaging (424-709/8.4-12 [TR/TE], 4.0-mm section thickness, and 0.4-mm intersection gap); axial T1-weighted imaging (519-745/6.8-12 [TR/TE], 3.0-mm section thickness, and 0.3$1.0-\mathrm{mm}$ intersection gap); sagittal T2-weighted imaging (3000-4301/92-120 [TR/TE], 4.0-mm section thickness, and 0.4-mm intersection gap) or sagittal 3D T2-weighted imaging (1300-1500/133-148 [TR/TE], 2.0-mm section thickness, and no intersection gap); and axial T2-weighted imaging (23724247/88-120 [TR/TE], 3.0-mm section thickness, and 0.3-1.0$\mathrm{mm}$ intersection gap). Axial and sagittal T1-weighted imaging were obtained within 10 minutes after IV administration of gadopentetate dimeglumine (Magnevist ${ }^{\circledR}$; Schering : 0.1 $\mathrm{mmol} / \mathrm{L}$ per kilogram of body weight). The imaging matrix was $512 \times 256$ or $448 \times 291$ for the agittal plane, $320 \times 293$ or 448 $\times 445$ for the 3D T2 sagittal plane, and $340 \times 253$ or $448 \times 260$ for the axial plane. The field of view was $240-300 \mathrm{~mm}$ depending on the patient's size and section planes. For the cervical spine, MRI was performed with a neck coil and using the same parameters in cases of lumbar or thoracolumbar spine, except for the image matrix, as follows : $448 \times 291$ for the sagittal plane, $320 \times 317$ for the 3D T2 sagittal plane, and $384 \times 174$ for the axial plane. The field of view was $200-380 \mathrm{~mm}$ depending on the patient's size and section planes.

Two musculoskeletal radiologists retrospectively interpreted the MRI findings in consensus regarding the postoperative spinal infection. Findings of postoperative spinal infection on MRI were evaluated as follows : 1) abscess or phlegmon in the 
paravertebral space, epidural space, laminectomy site, back muscles or any other sites appearing as low- to isointense signal on T1WI, high signal on T2WI, and peripheral rim or diffuse enhancement of adjacent soft tissue after administration of IV gadolinium; 2) osteomyelitis was considered when the vertebral body showed low signal intensity on T1WI and high signal intensity on T2WI or loss of definition between the vertebral body and the intervertebral disc space; and 3) discitis was considered when signal changes were noted in the intervertebral disc space on T1WI and T2WI with or without intervertebral disc space narrowing, and enhancement of these areas after administration of IV gadolinium ${ }^{1,6,7,13)}$.

Infection sites were categorized as three groups by anatomic location based on MRI : anterior part - prevertebral space, vertebral body, intervertebral disc space, paravertebral space, and anterior epidural space; posterior part - posterior epidural space, operative site such as laminectomy area, back/neck muscle, and subcutaneous tissue; and both parts. If only the posterior margin of the psoas muscle was involved and there was no involvement of the anterior part in a patient who underwent surgery at the lumbar level, we categorized this area as the posterior part. This classification by anatomic location reflected surgical approach and procedure.

\section{RESULTS}

Characteristics of patients' demographics and surgical procedures are summarized in Tables 1 and 2, respectively. All patients, except for one, underwent surgery using a posterior approach (Table 2). Infection part and MRI findings of each part are summarized in Table 3. 72.1\% (31/43) of the patients presented with posterior infection seen on MRI (Fig. 1). In the posterior part, abscess or phlegmon in the back muscle (93.0\%) is the most common finding seen on MRI. Ten patients were proven to have infection in both parts (Fig. 2), and two patients were proven to have anterior part infection (Fig. 3) on MRI. The relation between the types of surgical procedures and infection parts on MRI are summarized in Table 4. The clinical onset of each infection part and the relation between clinical onset and detailed site on MRI are summarized in Tables 5 and 6, respectively. Patients in the posterior and both parts groups showing clinically acute onset were $90.3 \%$, and $70 \%$, respectively (Table 5). Abscess or phlegmon in the back muscle and laminectomy site are the most common MRI findings in the patients with acute and late onset (Table 6).

Table 1. Demographic data of patients with postoperative spinal infection

\begin{tabular}{lr}
\hline & Value $(\mathbf{n}=43)$ \\
\hline Sex & $27(62.8)$ \\
Male & $16(37.2)$ \\
Female & \\
Age & \\
Range : 34-83 years & \\
Mean : 64 years & $30(69.8)$ \\
$>60$ years & \\
Risk factors & $5(11.6)$ \\
Obesity (body mass index >30) & $13(30.2)$ \\
Diabetes & $24(55.8)$ \\
Smoking & $9(20.9)$ \\
Cardiovascular disease & \\
\hline
\end{tabular}

Values are presented as number (\%)

Table 2. Operative characteristics of patients with postoperative spinal infection

\begin{tabular}{lc}
\hline & Value ( $\mathbf{n = 4 3 )}$ \\
\hline Approach & \\
Posterior & $42(97.7)$ \\
Combined anterior/posterior & $1(2.3)$ \\
Location & \\
Cervical & $2(4.7)$ \\
Thoracolumbar & $5(11.6)$ \\
Thoracolumbosacral & $1(2.3)$ \\
Lumbar & $21(18.8)$ \\
Lumbosacral & $14(32.6)$ \\
Procedure & \\
Uninstrumented posterior interbody fusion & $6(14.0)$ \\
Instrumented posterior interbody fusion & $11(25.6)$ \\
Instrumented anterior and posterior interbody fusion & $1(2.3)$ \\
Discectomy & $4(9.3)$ \\
Discectomy with posterior fusion & $1(2.3)$ \\
Posterior fusion & $9(20.9)$ \\
Decompression & $7(16.3)$ \\
Decompression with ISD & $4(9.3)$ \\
Indication & \\
Spinal stenosis & $30(69.8)$ \\
Disc herniation & $5(11.6)$ \\
Trauma or fracture & $4(9.3)$ \\
Metastasis & $3(7.0)$ \\
Removal of hardware & $1(2.3)$ \\
\hline
\end{tabular}

Values are presented as number (\%). ISD : interspinous process decompression device 
Follow-up MRI was performed in 12 of the posterior part group patients. The follow-up interval ranged from four to 118 days (mean, 26.3 days). In 50\% (6/12) of the patients, infection spread into the anterior part and consequently both parts be- came infected, as seen on follow-up MRI (mean, 38.2 days). In $16.7 \%(2 / 12)$ of the patients, the infection site was spread into the more anterior portion of the posterior part but still presented with posterior part infection (mean, 8 days). 33.3\%
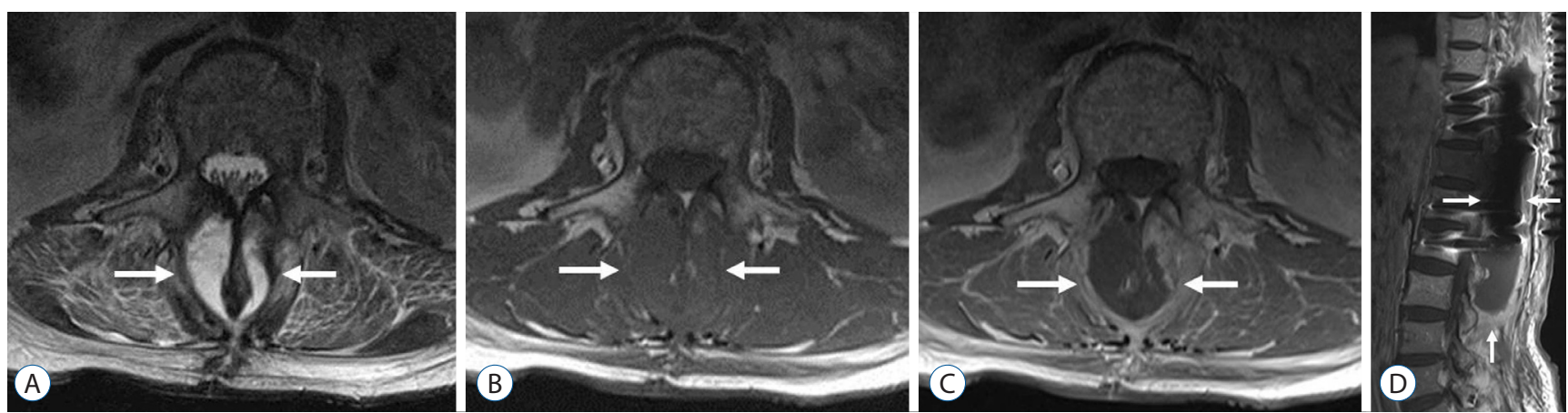

Fig. 1. A 54-year-old woman with postoperative spinal infection following excision of metastasis from a hepatoma involving T11, and posterolateral interbody fusion at T9-L1. Staphylococcus epidermidis infection was established through blood culture. A and B : Axial T2-weighted and T1-weighted images obtained 23 days following her operation showed a well-defined fluid collection (arrows) in the laminectomy site and in back muscles. C and D: Axial and sagittal images obtained after administration of intravenous gadolinium showed irregular and thick enhancement along the peripheral portion of the fluid collection (arrows), suggesting abscess formation in the laminectomy site and back muscles.
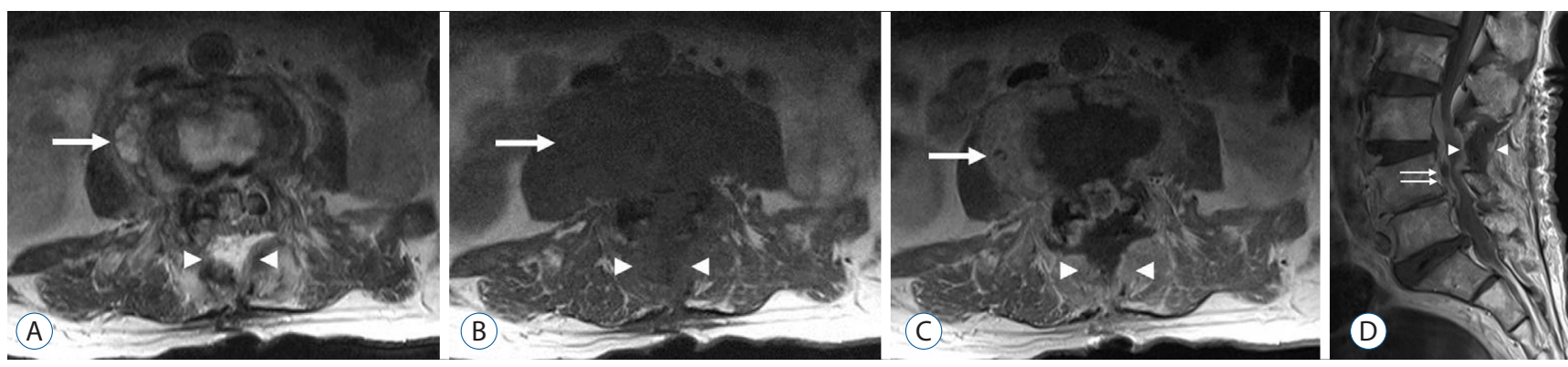

Fig. 2. A 67-year-old man with postoperative spinal infection following laminectomy at L3-4. Staphylococcus epidermidis infection was established through tissue biopsy. A and B : Axial T2-weighted and T1-weighted images obtained 48 days following his surgery showed a fluid signal-intensity lesion along the paravertebral (arrow) and laminectomy site (arrowheads). C and D : Axial and sagittal images obtained following administration of intravenous gadolinium showed osteomyelitis of L3 and L4 with paravertebral phlegmon (arrow), a laminectomy site (arrowheads), and epidural abscess (double arrow) with inflammation.
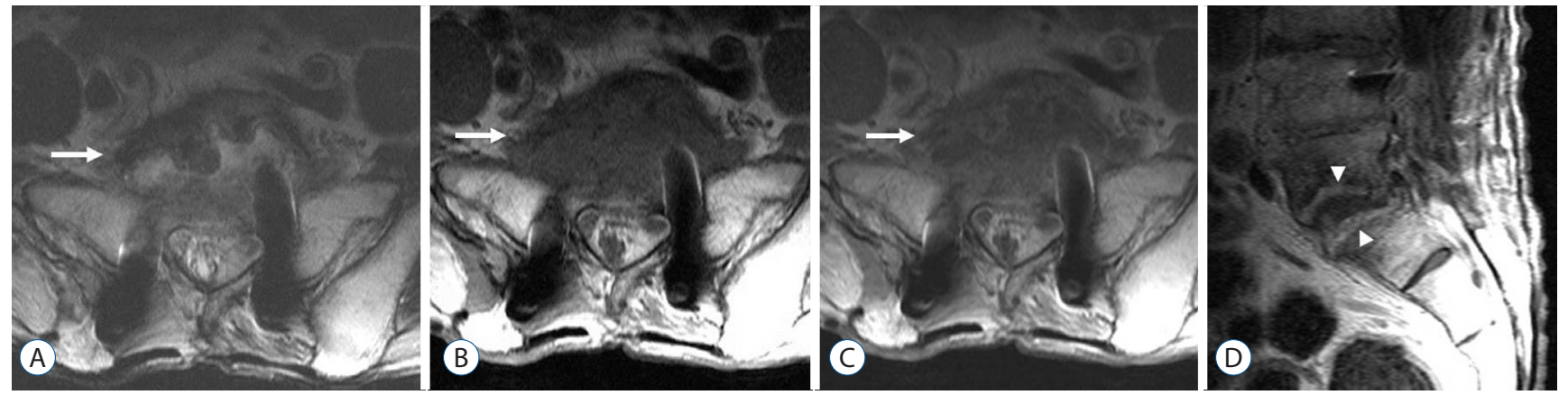

Fig. 3. A 62-year-old man with postoperative spinal infection following posterolateral interbody fusion with instrumentation at L3-S1. Pseudomonas aeruginosa infection was established through tissue biopsy. A and B : Axial T2-weighted and T1-weighted images obtained seven months after his surgery showed a paravertebral soft-tissue lesion (arrow) at L5-S1. C and D : Axial and sagittal images obtained after administration of intravenous gadolinium showed heterogeneous enhancement in paravertebral soft tissue (arrow) and along the endplate of the vertebral bodies (arrowheads) of $\mathrm{L} 5$ and S1. 
$(4 / 12)$ of the patients showed no change in the infection site (mean, 19.5 days).

The clinical onset ranged from one to 730 days with a mean value of 33.4 days following surgery. The mean durations from surgery to the clinical onset, from the clinical onset to MRI, and from surgery to MRI of each infection part are summarized in Table 7. Fever and a wound were the most common symptoms ( $n=19,19$, respectively). Back pain $(n=6)$, radiating pain $(n=2)$, and myalgia $(n=1)$ were also observed. ESR (mean, 72.45; range, 23-130) and CRP (mean, 9.80; range, 1.2-30.1) were high in all patients (100\%). WBC counts were elevated in 22 patients (51\%). The number of patients confirmed by positive cultures on aspirated pus, tissue biopsies, blood or cere-

Table 3. Infection part and relevant MRI findings of postoperative spinal infection

\section{Value $(n=43)$}

Infection part

$\begin{array}{lc}\text { Posterior } & 31(72.1) \\ \text { Anterior } & 2(4.7) \\ \text { Both } & 10(23.3) \\ \text { Imaging features } & \\ \text { Abscess/phlegmon in the posterior epidural space } & 14(32.6) \\ \text { Abscess/phlegmon in the laminectomy site } & 29(67.4) \\ \text { Abscess/phlegmon in the back muscle } & 40(93.0) \\ \text { Abscess/phlegmon in the subcutaneous fat layer } & 27(62.8) \\ \text { Abscess/phlegmon in the paravertebral space } & 9(20.9) \\ \text { Abscess/phlegmon in the psoas muscle } & 10(23.3) \\ \text { Osteomyelitis of the vertebral body } & 7(16.3) \\ \text { Discitis } & 7(16.3) \\ \text { Abscess/phlegmon in the anterior epidural space } & 9(20.9)\end{array}$

Values are presented as number (\%). MRI : magnetic resonance imaging brospinal fluid was 26, nine, six, and two, respectively. The causative micro-organisms are summarized in Table 8. Thirty-six patients underwent surgical treatment with either removal or retaining of instruments, and seven patients underwent conservative management.

\section{DISCUSSION}

Pull ter Gunne and Cohen ${ }^{17)}$ reported the mean time and the median time to the diagnosis of deep and superficial spinal infection. In their study, deep and superficial infection were defined by whether or not the infection extended beyond the fascia, whereas in our study, the location of the infection was determined based on MRI. This classification by anatomic location reflected surgical approach and procedure. Traditionally, postoperative spinal infections were classified into superficial or deep infection depending on the involvement of fascia. In the lumbar spine, deep infection occurred below the lumbodorsal fascia in posterior wounds and in the anterior abdominal fascia in anterior wounds. In the cervical spine, deep infection occurred below the ligamentum nuchae and the fascia layer in posterior wounds and in the platysma layer in anterior wounds ${ }^{3)}$. From the anatomic point of view, both the anterior and posterior parts were included in the deep infection, as seen on the traditional clinical view. In our study, only one patient presented with posterior infection limited to skin and the subcutaneous layer, and which is relevant to the superficial infection. In the surgical debridement of deep, postoperative spinal infection, meticulous eradication of the infection is important, whether the instrumentation is loose or in a well-fixed state. Therefore, it is essential for successful

Table 4. Relationship between type of surgical procedure and infection part on MRI

\begin{tabular}{lcccc}
\hline & Posterior & Both & Anterior & Total \\
\hline Uninstrumented posterior interbody fusion & $4(66.7)$ & $2(33.3)$ & $0(0.0)$ & $6(14.0)$ \\
Instrumented posterior interbody fusion & $7(63.6)$ & $4(36.4)$ & $0(0.0)$ & $11(25.6)$ \\
Instrumented anterior and posterior interbody fusion & $1(100)$ & $0(0.0)$ & $0(0.0)$ & $1(2.3)$ \\
Discectomy & $4(100)$ & $0(0.0)$ & $0(0.0)$ & $4(9.3)$ \\
Discectomy with posterior fusion & $0(0.0)$ & $1(100)$ & $0(0.0)$ & $1(2.3)$ \\
Posterior fusion & $6(66.7)$ & $1(11.1)$ & $2(22.2)$ & $9(20.9)$ \\
Decompression & $5(71.4)$ & $2(28.6)$ & $0(0.0)$ & $7(16.3)$ \\
Decompression with ISD & $4(100)$ & $0(0.0)$ & $0(0.0)$ & $4(9.3)$ \\
\hline Total & $31(72.1)$ & $10(23.3)$ & $2(4.6)$ & 43 \\
\hline
\end{tabular}

Values are presented as number (\%). MRI : magnetic resonance imaging, ISD : interspinous process decompression device 
patient management to decide whether the infection extends beyond the fascial plane as well as to identify any infectious foci before surgical treatment. In this respect, MRI has an important role regarding the evaluation of postoperative infection as it provides more accurate and detailed information regarding the infection site.

In general, infection is caused by inoculation at the time of surgery. The manipulation site is suitable for growth of bacteria owing to the injury of the endplate and small vascular structures and resultant hematoma and necrotic tissue formation during surgery ${ }^{11)}$. Moreover, back muscles and the subcutaneous fat layer, both of which were retracted during surgery, might be at risk for tissue ischemia, necrosis, and desiccation. These could increase the risk of wound contamination ${ }^{16)}$. According to the study by Pull ter Gunne and Cohen ${ }^{16)}$, the risk of surgical site infection showed a direct relationship to the surgical approach taken for spine surgery. In their study, the surgical site infection rate was significantly lower using the

Table 5. Clinical onset of each postoperative spinal infection part on MRI

\begin{tabular}{lcccc}
\hline Clinical onset & Posterior & Both & Anterior & Total \\
\hline Acute & 28 & 7 & 0 & 35 \\
Subacute & 1 & 1 & 0 & 2 \\
Late & 2 & 2 & 2 & 6 \\
Total & 31 & 10 & 2 & 43 \\
\hline
\end{tabular}

$\mathrm{MRI}$ : magnetic resonance imaging

Table 6. Relationship between clinical onset of postoperative spinal infection and detailed infection part on MRI

\begin{tabular}{lcccc}
\hline Infection part on MRI & $\begin{array}{c}\text { Acute } \\
(\mathbf{n}=\mathbf{3 5})\end{array}$ & $\begin{array}{c}\text { Subacute } \\
(\mathbf{n}=\mathbf{2})\end{array}$ & $\begin{array}{c}\text { Late } \\
(\mathbf{n}=6)\end{array}$ & $\begin{array}{c}\text { Total } \\
(\mathbf{n}=\mathbf{4 3})\end{array}$ \\
\hline $\begin{array}{l}\text { Posterior } \\
\quad \text { Posterior epidural space }\end{array}$ & $12(34.3)$ & $1(50)$ & $1(16.7)$ & $14(32.6)$ \\
Laminectomy site & $23(65.7)$ & $2(100)$ & $4(66.7)$ & $29(67.4)$ \\
Back muscle & $34(97.1)$ & $2(100)$ & $4(66.7)$ & $40(93.0)$ \\
Subcutaneous fat layer & $22(62.9)$ & $2(100)$ & $3(50)$ & $27(62.8)$ \\
Anterior & & & & \\
Paravertebral space & $5(14.3)$ & $1(50)$ & $3(50)$ & $9(20.9)$ \\
Psoas muscle & $7(20.0)$ & $2(100)$ & $1(16.7)$ & $10(23.3)$ \\
Vertebral body & $4(11.4)$ & $1(50)$ & $2(33.3)$ & $7(16.3)$ \\
Disc space & $3(8.6)$ & $1(50)$ & $3(50)$ & $7(16.3)$ \\
Anterior epidural space & $6(17.1)$ & $1(50)$ & $1(16.7)$ & $8(18.6)$ \\
\hline
\end{tabular}

Values are presented as number (\%). MRI : magnetic resonance imaging isolated anterior surgical approach than any in surgery that included a posterior surgical approach ( $1.7 \%$ vs. $4.4 \%)$. In our study, abscess or phlegmon in the back muscle (93.0\%), laminectomy site (67.4\%) and subcutaneous fat layer (62.8\%) are the most common MRI findings and this result might be explained by most operations were underwent using a posterior approach. A patient who underwent surgery using both anterior and posterior approaches, showed only posterior part (back muscle) infection on MRI. These findings are consistent with the results of the authors we mentioned. In our study, disc space manipulation such as cage insertion or discectomy were performed in 23 patients, which involving the both parts designated as anterior and posterior part. All patients showed posterior part infection and there were no anterior part infec-

Table 7. Mean duration of each part of postoperative spinal infection on MRI

\begin{tabular}{lccc}
\hline Mean duration (days) & Posterior & Both & Anterior \\
\hline Operation - clinical onset & 12 & 15.2 & 456.5 \\
Clinical onset - MRI & 7.5 & 17 & 130 \\
Operation - MRI & 19.5 & 32.1 & 495 \\
\hline
\end{tabular}

MRI : magnetic resonance imaging

Table 8. Micro-organisms isolated from culture in patients with postoperative spinal infection

\begin{tabular}{lc}
\hline Micro-organism & Value ( $\mathbf{n = 4 3 )}$ \\
\hline $\begin{array}{l}\text { Staphylococcus epidermidis } \\
\text { S. epidermidis }\end{array}$ & $19(20.9)$ \\
$\quad \begin{array}{l}\text { S. epidermidis+coagulase-negative } \\
\quad \text { Staphylococcus } \\
\text { S. epidermidis+Staphylococcus capitis }\end{array}$ & $1(16.3)$ \\
Methicillin-resistant Staphylococcus aureus & $1(2.3)$ \\
Methicillin-sensitive Staphylococcus aureus & $11(25.6)$ \\
Staphylococcus haemolyticus & $2(4.7)$ \\
Pseudomonas aeruginosa & $2(4.7)$ \\
Coagulase-negative Staphylococcus & $2(4.7)$ \\
Enterococcus faecalis & $1(2.3)$ \\
Enterococcus faecalis+coagulase-negative & $1(2.3)$ \\
Staphylococcus & $1(2.3)$ \\
Enterococcus faecium & $1(2.3)$ \\
Streptococcus agalactiae & $1(2.3)$ \\
\hline Escherichia coli & $1(2.3)$ \\
Peptostreptococcus micros & $1(2.3)$ \\
\hline
\end{tabular}

Values are presented as number (\%) 
tion without accompanied posterior part infection. All of 11 patients who underwent surgery involving only posterior part such as posterior decompression with or without interspinous process decompression device also showed same results. Moreover, $66.7 \%$ (8/12) of patients with posterior part infection showed anterior spreading pattern on the follow up MRI. According to these results, postoperative spinal infection undoubtedly occurred as a result of direct inoculation of the posterior part during surgery, and then undoubtedly spread into the anterior portion.

Pull ter Gunne et al. reported surgical site infection after spinal surgery occurred on average during 28.7 postoperative days (range, $5-730)^{17)}$. These findings are similar to our results. In our study, $86 \%$ (37/43) of the patients showed clinical symptoms and signs in less than four weeks following surgery, and $72.1 \%(31 / 43)$ of the patients were proven to have an abnormality seen on MRI within four weeks following surgery. In the study of Hamdan ${ }^{11)}$, the symptom of postoperative infection appeared between four days and three weeks following discectomy. In the study of Fang et al. ${ }^{9)}, 83.3 \%$ (40/48) of the postoperative deep wound spinal infections occurred within 90 days of surgery (range, 5-840 days). Therefore, careful follow-up is essential during the early postoperative period, especially within one month following spinal surgery, according to our study.

In our study, only six patients presented with late clinical onset, and in general, late onset infection was caused by lowvirulence pathogens ${ }^{5}$. These low-virulence organisms usually do not result in clinically significant sepsis, unless it is an immunocompromised patient ${ }^{4}$. Infection caused by a low-virulence pathogen was difficult to culture ${ }^{5)}$ and might have been excluded in our study, therefore resulting in a lower incidence. According to the study of Bose $\mathrm{e}^{2)}$, there were three possible mechanisms causing late infections : seeding of the surgical field during surgery; a sterile inflammatory reaction or stimulation of low-virulent organisms to fester caused by metal fretting; and hematogenous seeding. They reported four cases of delayed infection after spine surgery with instrumentation, two patients showed epidural or paraspinal abscess, and other patients showed a soft-tissue mass in the subcutaneous layer and fluid collection in the laminectomy site on computed tomography or MRI. They believed that late infection in their patients was caused by hematogenous seeding, due to the fact that all patients had a distant focus of infection before spinal infection. In our study, two anterior part cases presented with late onset infection. As these patients underwent posterior spine fusion using pedicle screw instrumentation, seeding of the surgical field during surgery could be excluded as the mechanism of infection. As the causative microorganisms in these patients were methicillin-resistant Staphylococcus aureus (MRSA), metal fretting could be excluded as the mechanism of infection. We could consider that these anterior infections might have been caused by hematogenous seeding, although none of the patients had a distant focus of infection.

It is well-known that Staphylococcus aureus is the most common causative organism and Staphylococcus epidermidis and $\beta$-hemolytic Streptococci are also commonly encountered pathogens ${ }^{14,15)}$. In our study, the most commonly detected microorganisms were Staphylococcus epidermidis $(\mathrm{n}=19)$ and MRSA ( $\mathrm{n}=11$ ). The contamination rate of Staphylococcus epidermidis in blood culture varied widely from as little as $0.6 \%$ to over $6 \%^{10)}$. Infection of Staphylococcus epidermidis occurred most commonly on intravenous catheters and on medical prostheses ${ }^{12}$. In our study, among the 19 patients who became infected with Staphylococcus epidermidis, 15 (78.9\%) had instruments used in their surgery and all of these patients improved after using antibiotics. Therefore, we considered that isolated Staphylococcus epidermidis in these cases was caused by instrumentation and was the true causative pathogen rather than contamination.

Our study had some limitations. First, as it was a retrospective study, all of the clinical information was obtained through review of electronic medical records. Second, as our medical institution is a tertiary referral center, the possibility that the culture results had been affected by previous antibiotic therapy before a patient's referral to our hospital, could not be excluded. Moreover, as we excluded culture-negative cases, there might have been a selection bias. Third, most operations were underwent using a posterior approach and these might affect infection sites. But as we have mentioned earlier, isolated anterior surgical approach is the factor decreasing infection risk, so there was much to be said for including a small number of anterior approach. Fourth, as the MRI findings of postoperative infection were investigated by radiologists and no controlled study was designed, some findings might have overlapped the normal postoperative findings. The last limitation is that a metal-induced susceptibility artifact might prohibit evaluating tissue surrounding a metallic implant on MRI. To 
reduce the metallic artifact, we obtained post-enhancement images without spectral fat saturation.

\section{CONCLUSION}

Postoperative spinal infection mainly occurred within four weeks after surgery in the posterior part and over time the infection was considered to spread into the anterior part, as seen on MRI. Abscess or phlegmon in the surgical area was the most common MR finding. Therefore, for the evaluation of postoperative spinal infection, on MRI, the posterior surgical field is more important than the anterior part such as vertebral body or the disc space.

\section{References}

1. Boden SD, Davis DO, Dina TS, Sunner JL, Wiesel SW : Postoperative diskitis: distinguishing early MR imaging findings from normal postoperative disk space changes. Radiology 184 : 765-771, 1992

2. Bose $B$ : Delayed infection after instrumented spine surgery: case reports and review of the literature. Spine J 3 : 394-399, 2003

3. Chaudhary SB, Vives MJ, Basra SK, Reiter MF : Postoperative spinal wound infections and postprocedural diskitis. J Spinal Cord Med 30 : 441-451, 2007

4. Clark CE, Shufflebarger HL : Late-developing infection in instrumented idiopathic scoliosis. Spine (Phila Pa 1976) 24 : 1909-1912, 1999

5. Collins I, Wilson-MacDonald J, Chami G, Burgoyne W, Vineyakam P, Berendt $T$, et al. : The diagnosis and management of infection following instrumented spinal fusion. Eur Spine J $17:$ 445-450, 2008

6. Djukic S, Genant HK, Helms CA, Holt RG : Magnetic resonance imaging of the postoperative lumbar spine. Radiol Clin North Am 28 : 341-
360, 1990

7. Djukic $S$, Vahlensieck $M$, Resendes $M$, Genant HK : The lumbar spine: postoperative magnetic resonance imaging. Bildgebung 59 : 136-146, 1992

8. Dufour V, Feydy A, Rillardon L, Redondo A, Le Page L, Bert F, et al. : Comparative study of postoperative and spontaneous pyogenic spondylodiscitis. Semin Arthritis Rheum 34 : 766-771, 2005

9. Fang $A$, Hu SS, Endres N, Bradford DS : Risk factors for infection after spinal surgery. Spine (Phila Pa 1976) 30 : 1460-1465, 2005

10. Hall KK, Lyman JA : Updated review of blood culture contamination. Clin Microbiol Rev 19 : 788-802, 2006

11. Hamdan TA : Postoperative disc space infection after discectomy: a report on thirty-five patients. Int Orthop $36:$ 445-450, 2012

12. Hedin G : Staphylococcus epidermidis--hospital epidemiology and the detection of methicillin resistance. Scand J Infect Dis Suppl 90 : 1-59, 1993

13. Kowalski TJ, Layton KF, Berbari EF, Steckelberg JM, Huddleston PM, Wald JT, et al. : Follow-up MR imaging in patients with pyogenic spine infections: lack of correlation with clinical features. AJNR Am J Neuroradiol $28: 693-699,2007$

14. Levi $A D$, Dickman CA, Sonntag VK : Management of postoperative infections after spinal instrumentation. J Neurosurg 86 : 975-980, 1997

15. Massie JB, Heller JG, Abitbol JJ, McPherson D, Garfin SR : Postoperative posterior spinal wound infections. Clin Orthop Relat Res (284) : 99108, 1992

16. Pull ter Gunne AF, Cohen DB : Incidence, prevalence, and analysis of risk factors for surgical site infection following adult spinal surgery. Spine (Phila Pa 1976) 34 : 1422-1428, 2009

17. Pull ter Gunne AF, Mohamed AS, Skolasky RL, van Laarhoven CJ, Cohen $\mathrm{DB}$ : The presentation, incidence, etiology, and treatment of surgical site infections after spinal surgery. Spine (Phila Pa 1976) 35 : 1323-1328, 2010

18. Van Goethem JW, Parizel PM, van den Hauwe L, Van de Kelft E, Verlooy J, De Schepper AM : The value of MRI in the diagnosis of postoperative spondylodiscitis. Neuroradiology 42 : 580-585, 2000 